\title{
Les chanoines de la cathédrale du Mans et le cumul des bénéfices au XIVe siècle (1294-1378)
}

Plurality of benefices and the canons of Le Mans Cathedral in the fourteenth century (1294-1378)

\section{Julien Leloup}

\section{OpenEdition}

\section{Journals}

Édition électronique

URL : http://journals.openedition.org/abpo/2563

DOI : $10.4000 / a b p o .2563$

ISBN : 978-2-7535-2782-9

ISSN : 2108-6443

\section{Éditeur}

Presses universitaires de Rennes

\section{Édition imprimée}

Date de publication : 30 mars 2013

Pagination : 33-58

ISBN : 978-2-7535-2780-5

ISSN : 0399-0826

\section{Référence électronique}

Julien Leloup, "Les chanoines de la cathédrale du Mans et le cumul des bénéfices au XIVe siècle (1294-1378) », Annales de Bretagne et des Pays de l'Ouest [En ligne], 120-1 | 2013, mis en ligne le 30 mars 2015, consulté le 19 avril 2019. URL : http://journals.openedition.org/abpo/2563 ; DOI : $10.4000 / a b p o .2563$ 


\title{
Les chanoines de la cathédrale du Mans et le cumul des bénéfices au XIV siècle (1294-1378)
}

\author{
Julien LELOUP \\ Enseignant au collège Jean-Rostand de Thouars
}

Depuis une trentaine d'années, les chapitres des cathédrales de France connaissent un regain d'intérêt auprès des chercheurs en histoire du Moyen $\widehat{A}_{g e}{ }^{1}$. L'historiographie des chapitres cathédraux remonte pourtant au XIX siècle. Mais, à cette époque, aucune étude ne portait sur les membres ordinaires du chapitre, ceux qui font sa vie quotidienne; les articles étaient essentiellement des études institutionnelles. Ainsi, en 1875, le chapitre cathédral du Mans a fait l'objet d'une étude qui couvre une période très large, de sa création à la Révolution de $1789^{2}$. Cette époque est aussi celle des publications de sources ${ }^{3}$.

Le renouvellement de l'historiographie des chapitres cathédraux est arrivé, en 1977, avec la thèse d'Hélène Millet qui est le premier travail universitaire sur les hommes du chapitre et non plus sur le chapitre lui-même ${ }^{4}$. C'est d'ailleurs à l'initiative et sous la direction de celle-ci que, dans les années 1990, s'est mis en place un groupe de recherche prospographique sur les membres des chapitres cathédraux de toute la France ${ }^{5}$. Celui de la

1. Cet article est issu d'un mémoire de master réalisé sous la direction de M. JeanMichel MATZ, professeur d'histoire du Moyen Âge à l'université d'Angers, intitulé Les chanoines du chapitre de la cathédrale Saint-Julien du Mans au XIve siècle (1294-1378). Étude d'un groupe social, mémoire de master 2 recherche d'histoire, université d'Angers, 2006.

2. BELLÉE, Armand, "L'ancien chapitre cathédral du Mans ", Bulletin de la Société d'Agriculture, Sciences et Arts de la Sarthe, Tome XxII, Le Mans, Monnoyer, 1875, p. 775-901.

3. Pour le chapitre cathédral du Mans voir par exemple BERTRAND de BROUSSILLON, Arthur et VAlléE, Eugène, Cartulaire de l'évêché du Mans, 936-1790, Le Mans, 1900 (Archives historiques du Maine, 1).

4. MiLLET, Hélène, Les Chanoines du chapitre cathédral de Laon, 1272-1412, Rome, École française de Rome, 1982.

5. Ce groupe de recherche publie les résultats de ses travaux par diocèse, dans le cadre de la collection Fasti Ecclesiae Gallicanae, éditée par Brepols Publisher, qui compte actuellement 12 volumes (Amiens, Rouen, Reims, Besançon, Agen, Rodez, Angers, Mende, Sées, Poitiers, Sens et Autun). 
cathédrale Saint-Julien du Mans n'a pas échappé à cet engouement avec de nouvelles études plus globales ${ }^{6}$, reprenant les mêmes problématiques et les mêmes méthodes qu'au niveau national. C'est dans cette lignée que se situe ce travail qui porte sur le XIV ${ }^{\mathrm{e}}$ siècle.

Cet article traite du cumul des bénéfices par les membres du chapitre entre 1294 et 1378, du début du pontificat de Boniface VIII à la fin de celui de Grégoire XI. L'année 1378 constitue une rupture tant dans l'Occident chrétien que dans la vie du chapitre cathédral car l'organisation de celui-ci est restée stable jusqu'à cette date ce qui n'est plus le cas ensuite.

La question du cumul a rarement été traitée en tant que telle. La plupart des travaux universitaires sur les chapitres cathédraux comportent une partie consacrée au cumul mais elle est rarement très développée et les articles concernant les bénéfices s'intéressent peu à ce sujet ${ }^{7}$. L'étude du cumul pose de nombreux problèmes car cette pratique a été pendant longtemps interdite; cela n'a cependant pas empêché les abus de se multiplier à la fin du XIII et au début du XIV siècle. Pour y faire face, le pape Jean XXII a réagi en promulguant la constitution Exsecrabilis, en 1317. Ce texte permet aux clercs, même dispensés, de disposer d'un seul bénéfice avec charge d'âmes (dignité, personnat, office, prieuré, église...) et d'un autre non grevé du soin des âmes ${ }^{8}$. Les clercs concernés par ces dispositions devaient résigner les bénéfices qu'ils possédaient en surplus, dans un délai d'un mois à compter de leur connaissance du décret. S'ils contrevenaient à ces dispositions, ils risquaient de les perdre et de ne pouvoir en obtenir d'autres ${ }^{9}$. En parallèle, une mainmise de la cour pontificale sur l'attribution des bénéfices s'est développée. En effet, la fin du XIII ${ }^{\mathrm{e}}$ siècle a vu la papauté s'impliquer de plus en plus dans la nomination aux bénéfices en général et aux dignités et canonicats des chapitres cathédraux en particulier. Les clercs sollicitant un bénéfice devaient fournir la liste de ceux qu'ils possédaient déjà, sous peine de les perdre. Cela permet de les connaître relativement bien et fait de l'usage des registres pontificaux un complément fondamental aux sources mancelles pour l'étude du cumul.

6. OGÉ, Nathalie, " Le chapitre cathédral du Mans aux XIII et XIV ${ }^{\mathrm{e}}$ siècles ", La Province du Maine, Le Mans, 1990, p. 249-258; Foullnet Thierry, Le chapitre cathédral du Mans et ses chanoines (1383-1448), mémoire de maîtrise d'histoire, université du Maine, Le Mans, 1998; Dupuy Charles-Antoine, Répertoire prosopographique des dignitaires et chanoines du chapitre cathédral Saint-Julien du Mans au XIIT siècle (1192-1294). Étude biographique d'un groupe canonial, mémoire de master 2 recherche d'histoire, université d'Angers, 2009.

7. TABBagh, Vincent, "Cumul des bénéfices ", dans VAuchez, André (dir.), Dictionnaire Encyclopédique du Moyen Âge, Paris, Le Cerf, 1997, tome I, p. 427 et 428. Cet article qui porte le titre de " cumul des bénéfices " est assez court et reste très général, il ne donne que de grandes orientations de la chronologie de la fin du Moyen Âge. Guillaume Mollat, dans ses nombreuses publications, n'est pas non plus très prolixe sur le sujet bien qu'il traite de la constitution Exsecrabilis et évoque assez succinctement la pratique du cumul.

8. Mollat, Guillaume, La collation des bénéfices ecclésiastiques sous les papes d'Avignon, Paris, E. de Boccard, 1921, p. 25.

9. Toutes ces prescriptions ne concernaient pas les cardinaux, ni les fils de roi. 
Dans la législation et dans les critiques qui en étaient faites, le cumul a souvent été vu sous un angle moral (enrichissement de quelques-uns). Le problème qui va nous intéresser ici revêt plutôt un aspect pratique : savoir quelles formes ce cumul de bénéfices a prises et en quoi le chapitre du Mans a pu en tirer profit ou au contraire en "souffrir". Cet article se penche donc aussi bien sur les chanoines cumulateurs que sur les différents types de bénéfices qu'ils ont cumulés et les conséquences du cumul pour le chapitre.

\section{Les chanoines cumulateurs}

$\mathrm{Au} \mathrm{XIV}^{\mathrm{e}}$ siècle, le chapitre du Mans était composé de neuf dignités (doyen ${ }^{10}$, maître-école, chantre et archidiacres du Mans ${ }^{11}$, de Sablé, de Laval, de Château-du-Loir, de Passais et de Montfort) et semblait compter quarante-trois prébendes. Cela ne correspond pas au nombre des membres du chapitre; le doyen, qui en est le chef, dispose en effet de deux prébendes et les abbés de Saint-Vincent du Mans et Saint-Calais ainsi que le roi de France ne sont membres que de nom. Il ne devait donc y avoir trente-cinq à quarante chanoines environ à la cathédrale du Mans à cette période, chaque dignité ou prébende du chapitre constituant un bénéfice.

Le corpus des cumulateurs du chapitre a été établi grâce aux sources pontificales qui ont été en grande partie éditées et complètent donc utilement les sources manuscrites ou imprimées disponibles au Mans ${ }^{12}$. Le travail, à partir des sources pontificales, pose cependant problème car certains chanoines disposant d'expectatives ${ }^{13}$ s'intitulent chanoines de telle cathédrale sans en être réellement membres. Des recoupements ont été aisés pour certaines cathédrales qui ont fait l'objet d'études pour la période concernée ${ }^{14}$. Pour les autres cas, les lettres pontificales précisent toujours les bénéfices en expectative; les bénéfices qui ne sont connus que

10. Le doyen était aussi archidiacre pour la ville du Mans et la Quinte (voir Annexe : Carte du diocèse du Mans).

11. L'archidiacre dit du Mans n'avait pas la ville du Mans dans sa juridiction car elle dépendait du doyen. Il contrôlait en fait le nord-est du diocèse, d'où son autre titre d'archidiacre de Saosnois.

12. Différentes personnes ont participé à ce travail pour publier les registres des pontificats de Boniface VIII (1294-1303), Benoît XI (1303-1304), Clément V (1304-1312), Jean XXII (1316-1334), Benoît XII (1334-1342), Urbain V (1362-1370) et Grégoire XI (1370-1378). Les registres de Clément VI (1342-1352) et Innocent VI (1352-1362) ont été partiellement publiés par l'Institut historique belge de Rome dans la collection Analecta-Vaticanobelgica.

13. Il était assez courant pour les clercs de recevoir des bénéfices dans des institutions alors qu'aucune bénéfice n'était disponible. La première place venant à se libérer leur revenait, en théorie, cependant certaines expectatives n'ont jamais été " transformées " en bénéfice réel.

14. Outre ceux qui ont fait l'objet d'études dans la collection Fasti Ecclesiae Gallicanae, les cathédrales de Laon, de Chartres et de Paris ont fait l'objet de thèses. Pour l'étranger les cathédrales anglaises ont toutes fait l'objet de volumes dans la collection Fasti Ecclesiae Anglicanae. 
par une expectative pendant le canonicat au Mans n'ont pas été pris en considération dans le corpus. C'est aussi le cas de personnages dont une expectative au Mans est connue sans que celle-ci soit confirmée par la suite. Grâce à ces documents, il a été dressé pour la période 1294-1378 une liste de 269 personnages, dont 118 cumulateurs, soit un peu moins de $40 \%$ des membres du chapitre ${ }^{15}$ qui disposaient en tout d'environ 300 bénéfices ${ }^{16}$.

\section{Origine sociale et géographique}

Les éléments de la vie des chanoines en dehors du chapitre sont assez peu connus, il est difficile d'en tirer des conclusions. Il est ainsi impossible de dire si le chapitre en général et les cumulateurs en particulier étaient plus ou moins ouverts à la noblesse. Les sources capitulaires ne sont pas très riches sur ce sujet même si des textes citent le nom de certaines grandes familles, comme les Beaumont ${ }^{17}$, ou de certains parents de chanoines pour qui ces derniers ont fondé des messes à la cathédrale. Les sources pontificales développent davantage ce point; certaines lettres donnent le nom et les fonctions du père du chanoine concerné. Certains textes parlent aussi de noble origine sans autre précision. On compte quatorze nobles et trois bourgeois (manceaux). Du fait de la faiblesse des données on ne peut tirer des conclusions de ces chiffres qui ne sont pas plus explicites pour le reste du chapitre. Il semble cependant que le cumul à grande échelle au début de la période soit principalement le fait de grandes familles (Bar, Beaumont, Périgord...) ${ }^{18}$.

L'origine géographique n'est pas mieux connue : sur 118 chanoines concernés, seuls 37 sont clairement identifiés. La première région à avoir fourni des chanoines est assez logiquement le diocèse du Mans, avec 16 personnes ${ }^{19}$. Certains personnages issus de quelques grandes familles mancelles sont assez faciles à identifier. On retrouve, au chapitre, des membres des familles Bouju, Béhuchet ou encore Riboul. Pour d'autres, la situation est plus difficile à cerner, les noms de lieux-dits, comme pour

15. En comparaison, à la cathédrale d'Angers, dans la deuxième moitié du XIV siècle, moins d'un quart des chanoines (37 sur 161) cumulait un autre bénéfice. BRION, Isabelle de, Les chanoines de la cathédrale d'Angers de 1356 à 1394, mémoire de maîtrise d'histoire, université d'Angers, 1996, p. 81.

16. Il y a en fait 301 bénéfices, dont une dizaine qui ne sont pas localisés. Ces statistiques ne prennent pas en compte les cardinaux. En effet, en vertu des dispositions règlementaires, ils possédaient une liste parfois extrêmement longue de bénéfices qui ne reflète pas du tout la situation commune.

17. Les Beaumont étaient vicomtes de Beaumont-du-Maine et formaient une très ancienne famille de la noblesse mancelle.

18. Archambault de Périgord, chanoine au Mans jusqu'en 1319, disposait de prébendes à Périgueux et à Paris, d'une abbaye, d'un prieuré et de l'archidiaconat de Dreux dans le chapitre de Chartres. Charles de Beaumont était chanoine de York, Salisbury et Lincoln. Louis de Beaumont était chanoine et trésorier de Salisbury, chanoine de Wells et de York.

19. À cette époque, le diocèse du Mans s'étendait sur haut et bas Maine, le diocèse de Laval ne datant que de 1855. 
Raoul de la Roche, ne permettent en effet pas de régler le problème. Pour deux personnages, Geoffroy de la Chapelle et Pierre de la Forêt, l'origine ne nous est connue que par leur testament. Les autres régions d'origine des chanoines sont ensuite les diocèses limitrophes de celui du Mans avec 10 clercs; 8 proviennent du pays des papes (Tulle, Limoges...) et deux d'Italie. Les chanoines du sud-est de la France ont profité des largesses des papes d'Avignon originaires de cette région, tout comme les deux Italiens, chanoines au début du XIV ${ }^{\mathrm{e}}$ siècle, qui ont profité de la bienveillance de Jean XXII et Clément V (1305-1314) et non de celle de Boniface VIII (1294-1303).

\section{Les cumulateurs : des gradués et des étudiants}

Du point de vue des études, le profil des chanoines cumulateurs est différent de celui du commun des chanoines, car les détenteurs de titres universitaires sont largement surreprésentés parmi eux ${ }^{20}$. Cela s'explique certainement par les lacunes de la documentation à ce sujet, surtout pour les sources capitulaires qui sont moins disertes que les sources pontificales. En tout cas, sur un total de 268 individus, 64 ont fait des études et parmi eux 55 sont cumulateurs. Les études semblent la conséquence du cumul; la plupart des chanoines ont obtenu leurs bénéfices après celles-ci. $97 \%$ des chanoines du Mans ont reçu leur prébende après avoir fréquenté l'université ce qui se rapproche de la situation d'autres chapitres comme Laon (75 \%). Cependant, certains chanoines ont dû se servir du cumul pour financer leurs études, comme Renaud Pansé qui quitte Le Mans pour faire des études à Toulouse après dix ans de résidence au chapitre ${ }^{21}$. Ces situations restent rares cependant.

Le nombre de diplômes est de 57 (la licence in utroque jure compte pour un); il est donc plus important que le nombre de personnes concernées et cela pour plusieurs raisons. Tout d'abord, on considère souvent la licence in utroque jure comme une seule catégorie de diplômés, or certains clercs gradués dans l'un et l'autre droit l'ont été successivement (cela concerne 19 diplômés). L'exemple le plus frappant est celui de Jean Le Verrier, chanoine au Mans de 1371 à 1381. Entre 1362 et 1371, il est successivement bachelier, licencié en lois puis étudiant et bachelier en droit canon. Finalement en 1371, il déclare être licencié in utroque jure. Par ailleurs, il existe des personnes graduées dans deux matières différentes à des niveaux différents (baccalauréat et licence par exemple), au Mans cela concerne 4 cumulateurs généralement plus gradués en droit civil qu'en droit canon.

20. Cependant le nombre total est certainement sous-évalué avec seulement $5 \%$ des chanoines du Mans gradués contre $50 \%$ au chapitre cathédral d'Angers à la fin du XIV ${ }^{\mathrm{e}}$ siècle. Cf. MATz Jean Michel, "La culture d'un groupe clérical, les chanoines de la cathédrale d'Angers, (milieu XIV ${ }^{\mathrm{e}}$-début XV ${ }^{\mathrm{e}}$ siècle) ", Revue d'Histoire de l'Église de France, tome 88, janvier-juin 2002, p. 24.

21. Bertrand de Broussillon, Arthur, Cartulaire..., op. cit., n 1125. 
Tableau 1 - Répartition des chanoines cumulateurs par grades et matières

\begin{tabular}{|c|c|c|c|c|c|c|c|}
\hline $\begin{array}{c}\text { Matière } \\
\text { Grade }\end{array}$ & Art & Médecine & Théologie & $\begin{array}{c}\text { Droit } \\
\text { canon }\end{array}$ & $\begin{array}{c}\text { Droit } \\
\text { civil }\end{array}$ & $\begin{array}{c}\text { Utroque } \\
\text { jure }\end{array}$ & TOTAL \\
\hline Maîtrise & 6 & 2 & & & & & 8 \\
\hline Baccalauréat & & & & 2 & 6 & 1 & 9 \\
\hline Licence & & & & 2 & 9 & 9 & 20 \\
\hline Doctorat & & & 2 & 1 & 6 & 7 & 16 \\
\hline Indéterminé & & & & 1 & 1 & 2 & 4 \\
\hline TOTAL & 6 & 2 & 2 & 6 & 22 & 19 & 57 \\
\hline
\end{tabular}

Au sein des études, le droit civil domine ainsi largement. Il représente 22 grades sur 57, contre 6 seulement pour le droit canon. Il n'y a que 6 titulaires de la maîtrise ès $\operatorname{arts}^{22}$. Le nombre de maîtres ès arts est naturellement le plus important étant donné que la maîtrise dans cette matière était nécessaire pour obtenir les diplômes suivants. Les catégories les moins représentées sont la médecine et la théologie avec chacune 2 représentants. Les études effectuées par les chanoines du Mans sont assez poussées, 20 licenciés dans leur matière et même 16 docteurs. Le reste des gradués se répartit également entre les maîtres et les bacheliers. Certains de ces personnages échappent à l'analyse; en effet, soit on connaît leur discipline d'étude et pas leur grade, soit on sait qu'ils ont été étudiants mais on ignore dans quelle discipline ${ }^{23}$.

La relative importance des gradués dans les personnes pratiquant le cumul s'explique, selon Hélène Millet, par l'intérêt porté par le pape aux diplômés ${ }^{24}$. Toujours selon cette auteure, le classement des diplômes est lié aux suppliants. Les institutions étatiques sont les premières servies dans les collations pontificales et essaient de placer les clercs de leur personnel $^{25}$. Les universités sont défavorisées face à ces administrations, or les diplômes de théologie et de droit canon n'étaient utiles que dans un cadre universitaire. Les gradués dans ces disciplines sont donc les " derniers servis " et reçoivent les bénéfices les moins intéressants. Certains chanoines, comme Jean Le Verrier, ont certainement préféré commencer par des études en droit civil avant de se lancer dans des études, peut-être moins rentables, en droit canon. Ces études leur permettaient de se mettre au service d'une " administration extérieure " pour obtenir ensuite leurs bénéfices.

22. Cette catégorie regroupe les individus qui sont nommés maître et ceux dont on connaît les diplômes de manière plus précise.

23. Renaud L'Usurier, chanoine de 1346 à 1349, est ainsi expert in utroque jure sans plus de précision.

24. Millet, Hélène, Les Chanoines de Laon..., op. cit., p. 186.

25. Ibidem, p. 188. 


\section{Des carrières dans les cours pontificale et royales}

Le service d'institutions extérieures est une affaire de cumulateurs : sur 58 chanoines qui ont eu une carrière extérieure identifiée, seuls 8 ne paraissent pas avoir cumulé plusieurs bénéfices.

Le plus grand pourvoyeur de cumulateurs au sein du chapitre cathédral est le pape ou la cour pontificale. Sur les 50 chanoines concernés, 26 sont au service de la curie, du pape ou de cardinaux. L'importance du groupe des proches de la curie est sans doute due à la mainmise progressive de la papauté sur la collation des bénéfices depuis le milieu du XIII ${ }^{\mathrm{e}}$ siècle. Il n'y a d'ailleurs aucun chanoine cumulateur et explicitement curialiste dans le chapitre avant le début du pontificat de Jean XXII. Cela tient peut-être aux sources mais aussi à la multiplication des cas de réserve qui ont maintenu un certain nombre de bénéfices dans les mains de la cour pontificale. D'autre part, les membres de la curie étaient plus à même de faire jouer leurs propres intérêts ou ceux de leurs serviteurs.

Dans les faits, le service pontifical recouvrait des réalités diverses. Le nombre de dénominations d'offices y était assez important et il est assez difficile de cerner la fonction précise de chacun; ainsi neuf chanoines sont intitulés chapelain pontifical. Si l'on en croit cette appellation, ces personnages sont au contact direct de la familia du pape mais cette titulature était parfois honorifique. Certains ont tout de même servi la chambre apostolique comme notaire ou auditeur des causes apostoliques. Le service des cardinaux est lui aussi assez difficile à cerner, les indications sur les activités étant assez rares, du fait du très large éventail de fonctions dans une familia cardinalice (du simple serviteur au secrétaire) ${ }^{26}$. Le vocabulaire employé est très varié et certains termes sont assez vagues comme celui de familier que l'on retrouve pour Nicolas de Lignon, proche du cardinal Guy de Boulogne. Pour d'autres, l'activité est plus nettement définie comme Pierre Syre (Tyre ou Coste), qui est le médecin du cardinal Hugues de SaintMartial $^{27}$.

L'intérêt du cumul pour la cour pontificale était d'assurer à ses membres un revenu sans en assumer la dépense. Ces cumulateurs étaient par ailleurs de bons relais de l'autorité du pape dans les différents diocèses concernés. Le chapitre en profitait aussi et disposait d'appuis à Avignon.

Les deuxièmes pourvoyeurs de chanoines cumulateurs sont les cours laïques. Le service du roi de France et de ses proches concerne 20 personnes. Les fonctions exercées par ces cumulateurs sont là aussi diverses et cela va de Guillaume de Feucherolles, " simple chapelain " de Philippe

26. JugiE, Pierre, « Les familiae cardinalices et leur organisation interne au temps de la papauté d'Avignon : esquisse d'un bilan ", dans Aux origines de l'État moderne : le fonctionnement administratif de la papauté d'Avignon, Rome, 1990 (Collection de l'École française de Rome, 138) p. 41-59.

27. Suppliques d'Urbain V, n 3601039; Archivum Secretum Vaticanum, Registre d'Avignon $\mathrm{n}^{\circ} 126, \mathrm{f}^{\circ} 306$. 
VI, à Pierre de la Forêt, chancelier de Jean II (1350-1364) ou Gilbert Hamelin, médecin de Charles IV (1322-1328) et de Philippe VI ${ }^{28}$. Certains chanoines, comme Martin de la Motte, sont simplement indiqués comme proches de tel ou tel roi, en l'occurrence Jean II. Il peut aussi s'agir enfin de membre du parlement de Paris comme Arnulphe Le Flament ${ }^{29}$. Le service du roi était la cause de la prébende et non pas la conséquence de qualités exceptionnelles exprimées au sein de la communauté, aucun chanoine ne semble avoir obtenu sa prébende au Mans avant d'entrer au service du roi. Elle est une récompense pour des services rendus. On retrouve d'ailleurs ce mécanisme dans le chapitre cathédral de Laon ${ }^{30}$. Les chanoines doivent faire leurs preuves au service d'un puissant avant de recevoir une prébende cathédrale.

Les frères et parents de rois ont aussi pourvu le chapitre cathédral du Mans de leurs serviteurs. Ils sont cependant moins nombreux et seuls deux chanoines sont concernés par le service d'autres seigneurs laïcs : Jean de Chambre et Jean de Tocco. Le premier (doyen du chapitre de 1314 à 1322) semble avoir été confesseur de Charles de Valois. Le second (archidiacre du Mans au début du XIV e siècle) a été médecin des rois de Sicile Charles II (1285-1309) et Robert I ${ }^{\mathrm{er}}$ (1309-1343). La collation de Jean de Chambre à la tête du chapitre cathédral du Mans s'explique sans doute par une volonté de Charles de Valois, alors comte du Maine, de placer un homme de confiance au sein du chapitre. Pour ce qui est de Jean de Tocco, je n'ai pas retrouvé la date de sa collation à l'archidiaconat mais sa première mention dans les sources remonte à $1307^{31}$. Sa présence au sein du chapitre s'explique très bien s'il y est entré avant la cession du Maine par les rois de Naples (1290). Dans le cas contraire, les rois de Naples voulaient absolument le placer au Mans, où ils avaient encore des appuis pour le faire accepter.

Peu de clercs serviteurs de laïcs échappent au service de la famille royale de France et de ses descendants, hormis Guy de Laval, clerc de Jean III, duc de Bretagne (1312-1341) ${ }^{32}$. Cependant, ce dernier, membre d'une puissante famille mancelle, n'avait certainement pas besoin de se mettre au service du duc de Bretagne pour obtenir des bénéfices.

Les intérêts de ces laïcs sont les mêmes que ceux du pape : assurer un revenu à leurs serviteurs et placer des "proches " dans les institutions ecclésiastiques locales pour en recevoir le soutien.

28. Lettres Communes de Jean XXII, Paris, E. de Boccard, ${ }^{\circ} 25740,46905,53611,53625$; GANE, Robert, Le Chapitre cathédral de Notre-Dame..., op. cit., p. 329.

29. HAYEZ, Anne-Marie, Lettres Communes de Grégoire XI, Rome, École française de Rome, n ${ }^{\circ}$ 32327; Fournier III, p. 457; BERTRAND de BRoussillon, Arthur, VAllÉE, Eugène, Cartulaire..., op. cit., $\mathrm{n}^{\circ} 864$ et $\mathrm{n}^{\circ} 1324$.

30. Millet, Hélène, Les Chanoines de Laon..., op. cit., p. 189.

31. Bertrand de Broussillon, Arthur, Vallée, Eugène, Cartulaire..., op. cit., n 1096.

32. Lettres Communes de Jean xxii..., op. cit., $\mathrm{n}^{\circ} 11183$. 


\section{Les autres carrières}

Les autres carrières hors du chapitre sont rares; ainsi le service de l'université est souvent lié au service des puissants. La fonction d'enseignant n'est clairement connue que pour quatre personnages : Jean de Tocco, Jean Dubois, Gilles Bellemère et Pierre Bertrand. En effet, certains chanoines apparaissent comme professeur mais le lieu d'enseignement n'est pas précisé. Jean de Tocco enseignait la médecine à l'université de Naples ${ }^{33}$; il a probablement été nommé à ce poste par les rois de ce lieu. Jean Dubois enseignait à Paris, Pierre Bertrand à Angers et Gilles Bellemère à Angers, ainsi qu'à Avignon (il était très proche du pape Grégoire XI dont il était le chapelain et commensal).

Les derniers " patrons", au service desquels se sont mis les chanoines cumulateurs du Mans, sont les évêques qui employaient cinq personnes. Dans cette catégorie, la plupart des personnes concernées sont soit vicaire soit official, mais pas nécessairement de l'évêque du Mans. On retrouve des serviteurs des évêques d'Angers mais aussi de celui d'Auxerre. Bien que peu représentés au sein du chapitre, certains de ces serviteurs d'évêque ont obtenu de nombreux bénéfices et même des dignités prestigieuses, comme Laurent Baulanié, doyen du chapitre et official du Mans. Il avait succédé à Robert Hélie, vicaire épiscopal et official d'Angers. Les chanoines au service d'évêques sont souvent originaires du Maine. La présence de Bertrand de Thierne, vicaire spirituel de l'évêque d'Auxerre, est certainement due à son office de conseiller au Parlement de Paris bien plus qu'à son vicariat ${ }^{34}$.

Le principe de la carrière extérieure au chapitre est réellement un point de différenciation des chanoines cumulateurs par rapport aux autres chanoines. Si tous les cumulateurs n'ont pas suivi cette voie, en revanche presque aucun des non-cumulateurs ne semble avoir eu de carrière extérieure. Le fait de se mettre au service d'un puissant a donc été, au XIV siècle, le meilleur moyen d'obtenir non seulement une prébende cathédrale mais plusieurs bénéfices à la fois. La prééminence pontificale constatée au Mans se retrouve dans d'autres chapitres, comme à Laon par exemple à la fin du XIV siècle $^{35}$. Chaque partie y trouvait un intérêt. Le chapitre profitait d'appuis dans ces institutions, tissant ainsi son réseau dans les « hautes sphères " de l'État royal ou pontifical. Les administrations faisaient porter sur d'autres le coût de leur fonctionnement.

\section{Répartition des bénéfices cumulés}

Les quelques 300 bénéfices cumulés par les membres du chapitre cathédral du Mans se répartissent en prébendes et dignités de cathédrales et de

33. WiCKERSHEIMER Ernest, Dictionnaire biographique des médecins en France au Moyen Âge, t. II, Genève, Droz, 1979, p. 493.

34. HAYEZ, Michel (dir.), Lettres Communes d'Urbain V..., Rome, École française de Rome, $\mathrm{n}^{\circ} 2319$.

35. MiLlet, Hélène, Les Chanoines de Laon..., op. cit., p. 190. 
collégiales ainsi que de nombreuses églises paroissiales, chapellenies et d'autres bénéfices moins nombreux (prieuré et abbaye) ${ }^{36}$.

\section{Une importante extension géographique}

Tous ces bénéfices sont répartis sur le territoire qui correspond à la France actuelle. On en retrouve aussi bien dans le diocèse de Thérouanne que dans ceux de Saint-Pol-de-Léon ou de Narbonne. Certains bénéfices sont en outre situés en Angleterre, en Italie ou en Belgique actuelle.

Le diocèse du Mans arrive assez largement en tête, avec 66 bénéfices, et devance celui d'Angers, avec " seulement " 43 bénéfices concernés. Ce total est malgré tout élevé si on le compare aux troisième et quatrième diocèses, Paris et Tours, avec respectivement 17 et 13 bénéfices chacun. Il existe donc de grandes disparités entre les diocèses : sur les 68 concernés par le cumul, 33 ne sont touchés que pour un seul bénéfice. La concentration la plus importante se situe dans la région à proximité du Mans. Si l'on regroupe les diocèses à l'est de la Bretagne jusqu'à ceux de Rouen, Paris et Sens, on retrouve $60 \%$ des bénéfices pour seulement $18 \%$ des diocèses. La majorité des bénéfices se trouve donc dans une région délimitée par la Seine, la Loire et la Bretagne.

La Bretagne semble échapper au cumul de la part des chanoines manceaux. Ceci n'est pas une exception locale car, comme l'a exposé Louis Caillet dans son étude de la politique bénéficiale de Jean XXII, les diocèses bretons paraissent plus récalcitrants que les autres à l'entrée de clercs extérieurs ${ }^{37}$.

Il existe des constantes entre les différents types de bénéfices, comme la proximité du diocèse du Mans; néanmoins il y a des différences dans la répartition géographique selon les bénéfices, qu'il s'agisse des prébendes cathédrales, des prébendes de collégiales ou enfin des autres bénéfices.

\section{La prédominance des bénéfices de chapitres cathédraux}

Les bénéfices les plus touchés par le cumul sont les prébendes de cathédrale avec 59 chanoines manceaux concernés ${ }^{38}$ dans 47 cathédrales essentiellement concentrées au nord de la Loire. Comparé à d'autres chapitres cathédraux, celui du Mans n'est malgré tout pas « hors norme ». À la fin du

36. Il y a en fait 292 bénéfices que j'ai réussi à localiser et une dizaine qui ne le sont pas, comme pour Pierre de Vallibus qui disposait de quatre bénéfices dans la province ecclésiastique de Tours sans autre précision.

37. CAILlET, Louis, La Papauté d'Avignon et l'Église de France, La politique bénéficiale de Jean XXII en France (1316-1334), Paris, PUF, 1975, p. 274, 302.

38. 35 chanoines disposent d'une seule prébende en plus de celle du Mans, 11 chanoines en cumulent deux et seulement 9 en cumulent 3 . Les cas les plus importants sont très minoritaires, 3 chanoines possèdent 4 prébendes et un en a 9 . Thibaud de Bar avait 10 prébendes (celle du Mans incluse) avec Paris, Lincoln, Metz, Liège, Troyes, Toul, Beauvais, Reims, Verdun et en plus une église paroissiale dans le diocèse de Chichester en Angleterre. 
Figure 1 - Répartition géographique des bénéfices cumulés par les chanoines de la cathédrale du Mans au XIV siècle

(d'après SINCLAIR, Stéphane, Atlas de géographie historique de la France et de la Gaule, de la conquête césarienne à nos jours, Paris, 1985, p. 190.)

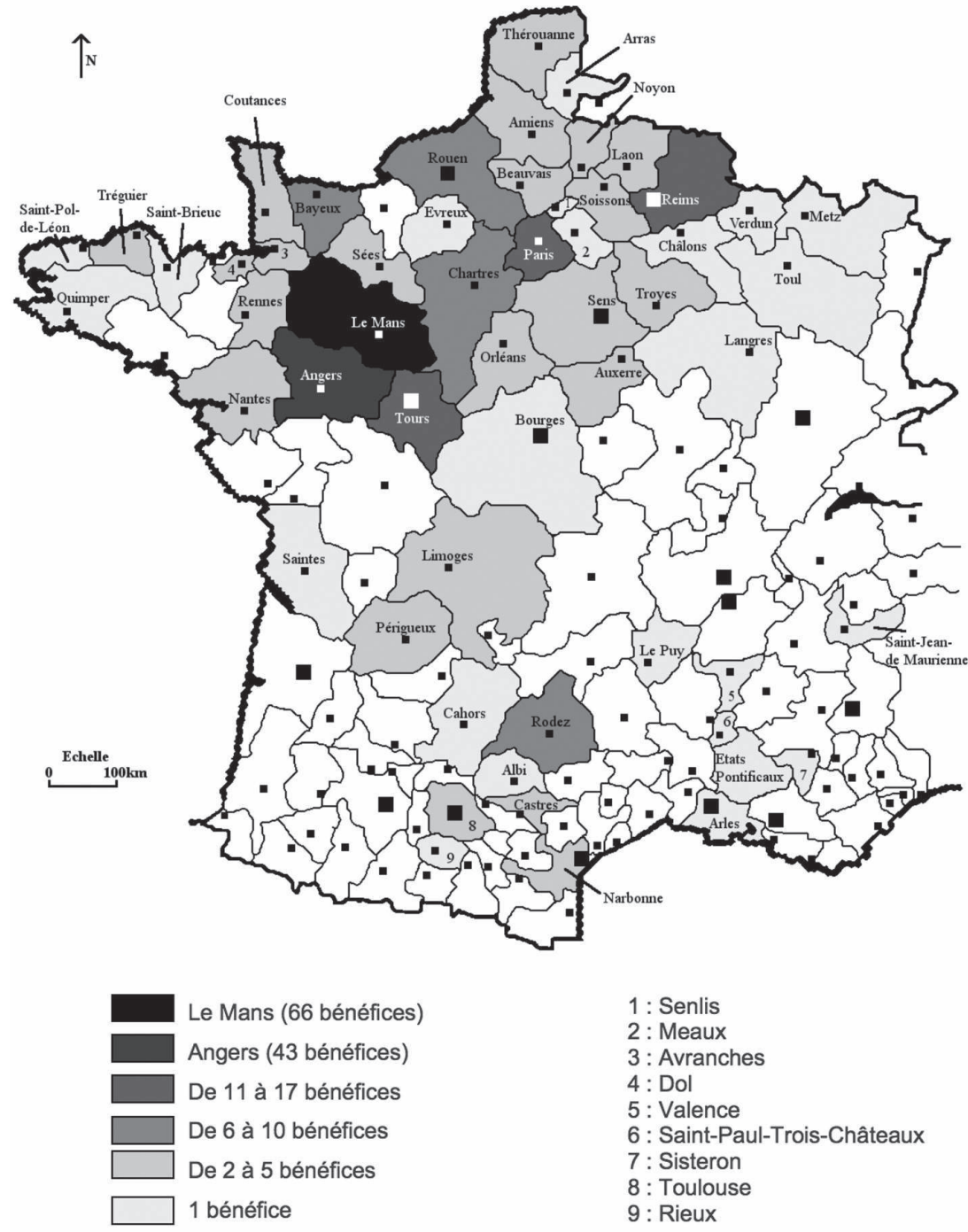

XIV ${ }^{\mathrm{e}}$ siècle, le chapitre d'Angers compte ainsi 25 chanoines cumulateurs de prébendes pour 43 bénéfices. La proportion entre le nombre de personnes et le nombre de bénéfices est équivalente ${ }^{39}$. En revanche, si l'on compare

39. BRIon, Isabelle de, Les Chanoines de la cathédrale d'Angers..., op. cit., p. 81. 
Figure 2 - Répartition géographique des bénéfices de cathédrales cumulés par les chanoines du Mans

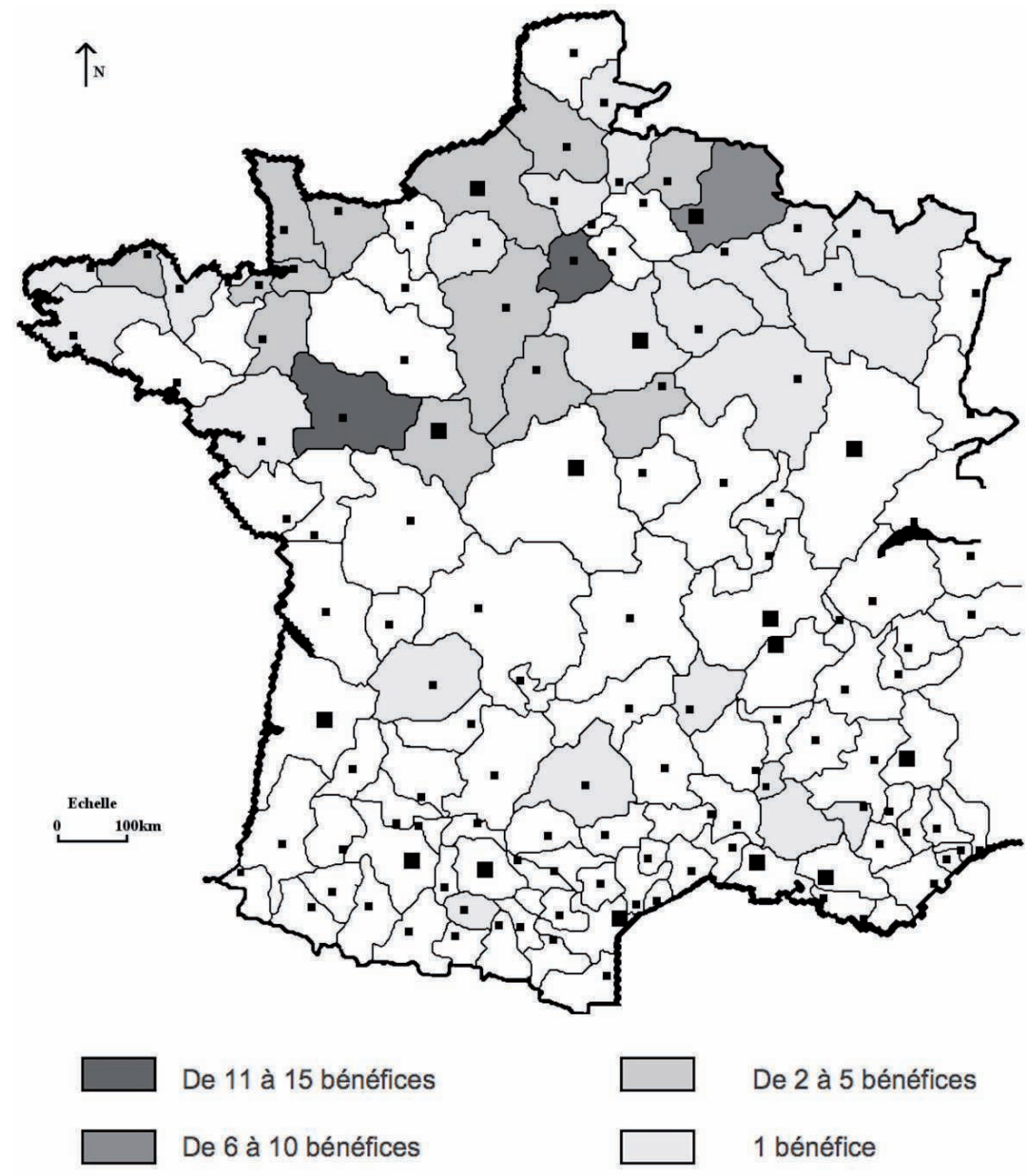

Le Mans à Notre-Dame de Paris, la différence est assez impressionnante : au XIV ${ }^{\mathrm{e}}$ siècle, les 87 chanoines cumulateurs de cette cathédrale ont plus de 250 prébendes, soit une moyenne de 2,8 par personne ${ }^{40}$.

Le chapitre cathédral d'Angers est celui dans lequel les chanoines du Mans ont cumulé le plus de bénéfices, et cela se retrouve tout au long de la période. Cette présence s'explique aisément par une proximité géographique mais aussi politique. Depuis le XII ${ }^{\mathrm{e}}$ siècle les comtes d'Anjou

40. Gane, Robert, Le Chapitre cathédral de Notre-Dame de Paris au XIVe siècle, Étude sociale d'un groupe canonial, Saint-Étienne, Publications de l'université de Saint-Étienne, 1999, p. 109. 
Figure 3 - Répartition géographique des bénéfices de collégiales cumulés par les chanoines du Mans

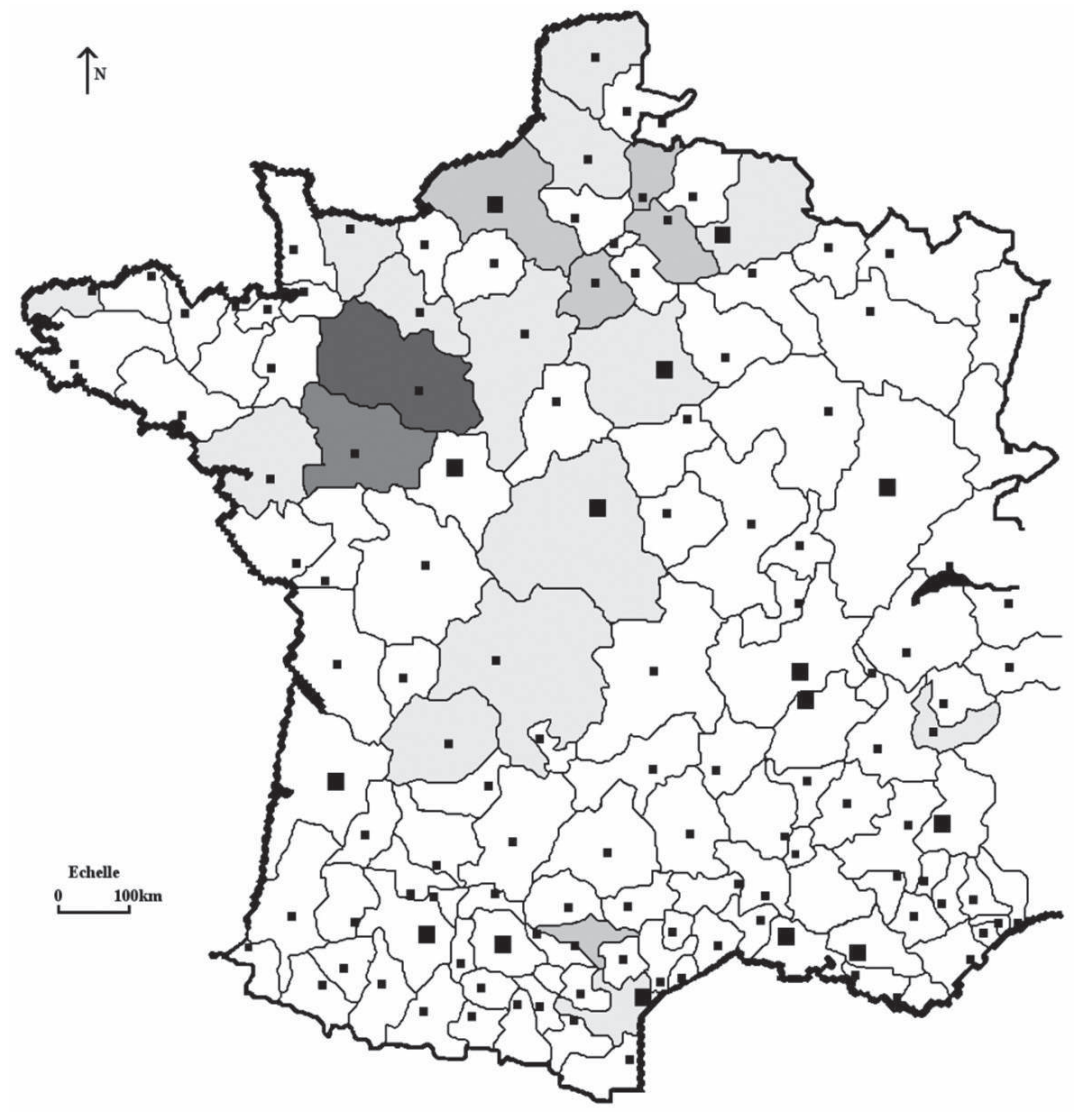

(Plantagenêts ou Capétiens) sont maîtres du Maine, ils ont donc placé de longue date des membres de leur entourage dans les deux chapitres cathédraux. 
Tableau 2 - Nombre total de bénéfices relevant de chapitres cathédraux détenus par des chanoines du Mans (par diocèse)

\begin{tabular}{|c|c|c|c|}
\hline Diocèse & $\begin{array}{l}\text { Nombre de } \\
\text { bénéfices }\end{array}$ & Diocèse & $\begin{array}{l}\text { Nombre de } \\
\text { bénéfices }\end{array}$ \\
\hline Angers & 15 (dont 3 dignités) & Châlons & 1 \\
\hline Paris & 11 & Évreux & 1 \\
\hline Reims & 6 (dont 1 dignité) & Langres & 1 \\
\hline Chartres & 5 (dont 1 dignité) & Le Puy & 1 dignité \\
\hline Rouen & 5 (dont 1 dignité) & Metz & 1 \\
\hline Laon & 4 & Nantes & 1 \\
\hline Orléans & 4 (dont 2 dignités) & Noyon & 1 \\
\hline Tours & 4 (dont 3 dignités) & Palerme & 1 \\
\hline Dol & 3 & Périgueux & 1 \\
\hline Bayeux & 3 (dont 2 dignités) & Quimper & 1 \\
\hline Coutances & 3 (dont 1 dignité) & Rieux & 1 \\
\hline Amiens & 2 & Saint-Brieuc & 1 dignité \\
\hline Avranches & 2 & $\begin{array}{l}\text { Saint-Paul-Trois- } \\
\text { Châteaux }\end{array}$ & 1 dignité \\
\hline Auxerre & 2 & Saint-Pol-de-Léon & 1 \\
\hline Liège & 2 & Sens & 1 \\
\hline Lincoln & 2 & Sisteron & 1 \\
\hline Rennes & 2 (dont 1 dignité) & Toul & 1 \\
\hline Salisbury & 2 (dont 1 dignité) & Tournai & 1 \\
\hline York & 2 & Tréguier & 1 dignité \\
\hline Arras & 1 & Troyes & 1 \\
\hline Avignon & 1 dignité & Verdun & 1 \\
\hline Beauvais & 1 & Volterra & 1 \\
\hline Bologne & 1 & Wells & 1 \\
\hline & & TOTAL & $\begin{array}{l}107 \\
\text { (dont } 21 \text { dignités) }\end{array}$ \\
\hline
\end{tabular}

Un autre élément important découle des deux précédents, la confraternité entre les deux chapitres. Aux environs de 1244, il semble qu'un accord de confraternité ait été signé qui prévoyait la prière dans chaque chapitre pour les défunts de l'autre, mais aussi l'entraide en cas de problème ${ }^{41}$. Le nombre de chanoines " manceaux-angevins " peut enfin s'expliquer par la présence à Angers d'une université mais en l'absence de liste complète des étudiants, rien ne prouve que la prébende angevine ait constitué une bourse d'étude.

41. RENOUARD, Pierre, Essais historiques et littéraires sur la ci-devant province du Maine, divisés par époque, Le Mans, Fleuriot, 1811, p. 293. Cet accord a été réaffirmé au XV siècle (Bib. mun. d'Angers, ms. 724). 
En dehors d'Angers, les chapitres des cathédrales du Nord-Ouest (hors Bretagne) représentent $45 \%$ des bénéfices pour seulement $20 \%$ des diocèses. Les seuls diocèses à échapper à la règle sont ceux de Sées et de Lisieux ${ }^{42}$.

La proximité géographique apparaît comme une des principales causes du cumul dans la plupart des diocèses comme Chartres, Orléans ou Tours. D'autres éléments ont pu s'y ajouter pour renforcer l'attractivité de certaines cathédrales; Paris tire ainsi son importance de la proximité avec le pouvoir royal et l'université. D'après Hélène Millet qui a étudié le cumul chez les chanoines dans la base de données Fasti, Paris a les faveurs de tous les cumulateurs de prébendes cathédrales des diocèses du nord de la Loire $^{43}$. Le chapitre de Reims est lui aussi très prestigieux à cause du sacre des rois de France, ce qui compense son éloignement géographique. Dans le cas de Rouen, la proximité relative a été probablement renforcée par le prestige de la cathédrale archiépiscopale. En effet, la plupart des chanoines ont obtenu leur bénéfice rouennais après leur prébende mancelle. Par ailleurs, aucune autre relation ne semble lier les deux chapitres bien qu'ils aient été tous les deux sous la domination des rois d'Angleterre. En ce qui concerne la cathédrale de Laon, le nombre de chanoines manceaux n'est en fait pas très élevé par rapport aux 92 prébendes et aux 843 chanoines relevés entre 1272 et $1412^{44}$.

Pour les autres chapitres cathédraux de France, la faiblesse du nombre de chanoines est donc souvent liée à l'éloignement géographique, mise à part la Bretagne évoquée plus haut. Le cumul dans des diocèses de l'est de la France (Metz, Verdun et Toul) s'explique simplement par la présence au Mans de Thibault de Bar, originaire de cette région, et par les appuis de sa famille en Lorraine. Pour ce qui est de la prébende mancelle, les relations sont plus lointaines ${ }^{45}$ et une motivation financière n'est pas à exclure. La recherche du prestige au Mans paraît un peu secondaire.

Le cumul de prébendes et de dignités dans des chapitres cathédraux du Sud s'explique probablement par l'appui du pape pour l'obtention d'une prébende en France du Nord. En effet, les diocèses situés aux alentours

42. Le chapitre de Sées échappe au cumul car il est resté régulier jusqu'en 1547 et la possession d'une prébende en son sein était donc plus contraignante et plus réglementée. DesPortes Pierre, Foucher Jean-Pascal, LodDÉ Françoise, VALLIĖRE Laurent, Fasti Ecclesiae Gallicanae, volume 9, Le diocèse de Sées, Turnhout, Brepols, 2005, p. 8-11.

43. Les chapitres d'Amiens, de Rouen ou de Reims se tournent vers ce chapitre avant même ceux de leur province ecclésiastique. MiLLET, Hélène, "Les Fasti Ecclesiae Gallicanae ", dans VACCARO, Luciano (éd.), Storia della Chiesa in Europa tra ordinamento politico-amministrativo e strutture ecclesiastiche, Brescia, École française de Rome et Fondazione Ambrosiana Paolo VI, 2005, p. 73-86.

44. Millet, Hélène, Les Chanoines de Laon..., op. cit.

45. Viton DE SAINT-AlLaIS, Nicolas, L'art de vérifier les dates des faits historiques..., tome XIII, Paris, Valade, 1818, p. 114-115. Thibault est le petit-fils de dame Emma de Laval (par sa mère, Jeanne de Toucy) et est donc cousin avec les seigneurs de Laval de l'époque, qui descendent du deuxième mariage d'Emma avec Mathieu II de Montmorency. Il est donc le cousin de Guy de Laval, chanoine du Mans, à peu près à la même période. 
des États pontificaux sont " peuplés " de membres de l'administration pontificale : les clercs bénéficiers de ces régions étaient à même d'exposer leurs demandes de manière rapide et en personne à la cour d'Avignon. Le Sud-Ouest est la région d'origine des papes du XIV ${ }^{\mathrm{e}}$ siècle et cela a profité aux clercs de cette région qui ont pu obtenir des bénéfices ailleurs dans la Chrétienté en général, et au Mans, en particulier. Le revenu d'un bénéfice des petits diocèses du Sud n'était sans doute pas comparable avec celui d'un chapitre de France du Nord. La preuve en est qu'un archidiacre de Saint-Paul-Trois-Châteaux devient chanoine de la cathédrale du Mans ${ }^{46}$. Par ailleurs, aucun de ces chanoines n'a reçu sa prébende du Mans avant celle dans le Sud de la France. La prébende mancelle apparaît donc d'un intérêt supérieur dans une carrière ecclésiastique. Il est probable que pour ces clercs n'importe quel chapitre cathédral de France du Nord aurait convenu, Le Mans n'ayant pas un prestige nettement supérieur à ses voisins angevin ou tourangeau, même si le roi en était membre.

Parmi les diocèses étrangers, l'Angleterre (Lincoln, Salisbury et York) est le territoire le plus concerné, avec la présence de Louis et Charles de Beaumont ${ }^{47}$. Louis de Beaumont, fils du vicomte de Beaumont, a obtenu une prébende à Salisbury par l'entremise du roi d'Angleterre Édouard I ${ }^{\mathrm{er}}$, en 1290. Cependant il n'a pas dû beaucoup résider dans ses bénéfices anglais. En effet, des sources locales précisent qu'il est " à l'étranger " en 1291 et 1299 et il est même rappelé à l'ordre en 1300 et 1303 par le chapitre de Salisbury, qui le menace de représailles ${ }^{48}$. La présence des Beaumont en Angleterre n'est pas étonnante car ils ont le soutien du roi d'Angleterre, descendant des Plantagenêts qui étaient jusqu'au début du XIII ${ }^{\mathrm{e}}$ siècle les comtes du Maine. La famille de Beaumont a donc profité de ses relations anciennes avec la famille royale d'Angleterre pour placer ses enfants, Louis et Charles, qui n'ont pas d'autres prébendes en dehors du Mans et de l'Angleterre. Le cumul de prébendes anglaises disparaît avec ces personnes, certainement du fait de la guerre entre la famille royale de France et celle d'Angleterre. Les autres diocèses étrangers sont nettement moins concernés ${ }^{49}$. Étonnamment, le cumul de prébendes italiennes a été possible grâce aux papes d'Avignon et non durant le pontificat de Boniface viII, pourtant souvent plus favorable aux Italiens dans d'autres chapitres ${ }^{50}$. Les trois personnes concernées sont des proches de la curie pontificale ${ }^{51}$.

46. Il s'agit d'Adémar Boeti chanoine du Mans en 1363, originaire du diocèse de Tulle (HAYEZ, Michel (dir.), Lettres Communes d'Urbain V..., $\mathrm{n}^{\circ}$ 6927).

47. Charles et Louis sont peut-être frères; cette idée est proposée par Diana Greenway dans : GREENWAy Diana Eleanor, Fasti Ecclesiae Anglicanae (1066-1300), tome IV, Salisbury, Londres, Institute of Historical Research, 1991, p. 121.

48. Ibidem, p. 23, 54, 59, 121.

49. Pierre de La Trémoille, archidiacre de Montfort jusqu'en 1374, est ainsi chanoine de Tournai en 1371.

50. À Laon, les Italiens sont très présents dans le chapitre sous le pontificat de Boniface VIII. MiLlEt Hélène, Les Chanoines de Laon..., op. cit., p. 62.

51. Hugues Le Boeuf était chanoine de Volterra et chapelain du cardinal Arnaud de Falguerris. Cinthius Arlotti de Urbe, quant à lui, était chanoine de Palerme et familier d'un 


\section{Les bénéfices de collégiales « locales»}

En dehors des cathédrales, pour un quart les bénéfices cumulés par 47 chanoines de la cathédrale du Mans sont des bénéfices de collégiales ${ }^{52}$.

Tableau 3 - Nombre total de bénéfices relevant de collégiales détenus par des chanoines du Mans (par diocèse)

\begin{tabular}{|l|l|l|l|}
\hline \multicolumn{1}{|c|}{ Diocèse } & Nombre de bénéfices & \multicolumn{1}{c|}{ Diocèse } & Nombre de bénéfices \\
\hline Le Mans & 13 (dont 3 dignités) & Chartres & 1 \\
\hline Tours & 9 (dont 4 dignités) & Gênes & 1 \\
\hline Angers & 8 (dont 1 dignité) & Liège & 1 \\
\hline Noyon & 4 & Nantes & 1 \\
\hline Paris & 4 & Narbonne & 1 \\
\hline Bayeux & 2 & Périgueux & 1 \\
\hline Castres & 2 & Reims & 1 \\
\hline Limoges & 2 & Rome & 1 \\
\hline Rouen & 2 dignités & $\begin{array}{l}\text { St-Jean-de- } \\
\text { Maurienne }\end{array}$ & 1 \\
\hline Soissons & 2 & Sées & 1 \\
\hline Tournai & 2 & Sens & 1 \\
\hline Amiens & 1 & Thérouanne & 1 dignité \\
\hline Barletta & 1 & York & 1 \\
\hline Bourges & 1 & TOTAL & 66 (dont 11 dignités) \\
\hline & & &
\end{tabular}

Les collégiales du diocèse du Mans (Saint-Pierre-la-Cour et Saint-Lazare au Mans, Notre-Dame de Laval et Saint-Pierre de Saint-Calais) sont les plus représentées. Le premier établissement est à la fois le plus concerné et le plus proche géographiquement. La collégiale Saint-Pierre-la-Cour se situe, en effet, à quelques centaines de mètres de la cathédrale. Huit chanoines de cette communauté sont aussi chanoines de la cathédrale. Cependant il est assez difficile d'établir clairement s'ils étaient membres des deux églises à la fois ou s'ils avaient abandonné la prébende de Saint-Pierre. L'étude produite par Menjot d'Elbenne liste les chanoines et dignitaires de SaintPierre-la-Cour mais se limite aux sources mancelles; le résultat final est certainement incomplet pour les dates des canonicats ${ }^{53}$.

autre cardinal. Enfin, dans l'expectative d'Arnaldus de Asiniis (chanoine de Bologne) pour Le Mans, le cardinal-évêque d'Ostie est cité.

52. Guillaume de Feucherolles est le cas le plus impressionnant avec pas moins de cinq prébendes répertoriées! Cela est certainement la conséquence de son service envers le roi Philippe VI (1328-1350).

53. Menjot d'Elbenne, Samuel, Le Chapitre royal de l'église collégiale de Saint-Pierre-laCour, Sainte-Chapelle du Mans, Le Mans, 1909 (Archives historiques du Maine, 10). Ce travail a été fait essentiellement à partir des sources sarthoises et très peu à partir des sources pontificales, ou tout au moins pas de façon systématique. 
Ceci étant, il apparaît que le choix de la cathédrale par rapport, ou en concomitance, avec sa voisine, s'est certainement fait pour des motivations financières, tout autant que prestigieuses, car certaines personnes apparaissent dans les deux listes ${ }^{54}$. À titre de comparaison, dans un compte édité par Auguste Longnon, les chanoines de la cathédrale paient tous, à la cour pontificale, un impôt de 30 sous au titre des décimes, à l'opposé de ceux de Saint-Pierre-la-Cour qui ne sont imposés qu'à hauteur de 20 sous, ce qui est à peine plus élevé que les chapelains de la cathédrale (de 16 à 40 sous $)^{55}$. Les autres collégiales du diocèse sont nettement moins touchées : un ou deux chanoines tout au plus cumulent des prébendes dans celles-ci. Ces collégiales sont fréquemment la dernière étape avant l'obtention d'une prébende cathédrale, le plus souvent dans leur diocèse d'origine. Dans ce contexte, c'est souvent la proximité géographique qui doit entrer en ligne de compte mais il faudrait étudier les autres chapitres cathédraux pour savoir si des chanoines de collégiales mancelles ont obtenu des prébendes dans d'autres cathédrales de France.

Le diocèse du Mans devance de peu celui de Tours, dont la deuxième place s'explique par le prestige de la collégiale Saint-Martin qui regroupe à elle seule la quasi-totalité des prébendes cumulées dans le diocèse ${ }^{56}$. L'importance d'Angers tient probablement aux mêmes raisons que pour les prébendes cathédrales. Les autres diocèses sont assez peu concernés par ce type de cumul. Noyon constitue un cas un peu particulier, avec quatre bénéfices pour la seule collégiale de Saint-Quentin qui dispose d'un prestige aussi important que n'importe lequel des chapitres cathédraux du Nord de la France, selon Pierre Desportes ${ }^{57}$. Pour ce qui est des diocèses étrangers, l'Italie du Sud n'est présente que du fait de la prébende de Jean de Tocco à Saint-Jacques de Barletta, sa présence au Mans s'expliquant par sa fonction de médecin des rois de Naples et comtes du Maine. La prébende cathédrale du Mans représente, pour la plupart des possesseurs de prébendes collégiales, l'accomplissement de leur carrière, sauf pour ceux qui sont devenus évêques ensuite.

54. Henri de Dijon, Guillaume Mégret par exemple.

55. Longnon, Auguste, Pouillés de la province de Tours, Paris, 1903, Recueil des historiens de la France, p. 58.

56.7 des 9 bénéfices détenus dans des collégiales du diocèse de Tours, le sont à SaintMartin.

57. Ces quatre chanoines sont des proches du roi de France. Cela a un lien avec leur bénéfice en Vermandois car le roi disposait de plein droit de la nomination de prébendes de cette collégiale. DesPoRTEs, Pierre, "Saint-Quentin en Vermandois : la collégiale et la ville ", dans Le Bourgeors, Roselyne, Massoni, Anne, Montaubin, Pierre (dir.), Les Collégiales et la ville dans la province ecclésiastique de Reims, Amiens, Publications du CAHMER, 2010, p. 154. 


\section{Les autres bénéfices}

Outre les prébendes cathédrales et collégiales, d'autres bénéfices sont aussi représentés comme les églises paroissiales (24\% des bénéfices cumulés, 55 personnes concernées).

Rares sont les chanoines qui cumulent plusieurs églises paroissiales, pratique interdite en vertu de la constitution Exsecrabilis de 1317, prévoyant que les bénéfices ne sont pas cumulables avec d'autres bénéfices à charge d'âmes. Cette disposition ne semble cependant pas avoir arrêté les 14 personnes qui ont disposé de plus d'une église paroissiale ${ }^{58}$. Si on compare le chiffre manceau à celui d'Angers, la proportion de cumulateurs d'églises paroissiales est, à peu près, équivalente. Dans celui-ci, le cumul d'églises paroissiales et de chapellenies confondues intéresse seulement 14 chanoines pour 19 bénéfices ${ }^{59}$, soit une personne sur dix et un cumulateur sur deux environ.

Il n'y a que 20 diocèses tant français qu'étrangers qui sont touchés par ce cumul. Les écarts sont donc impressionnants; ainsi le diocèse du Mans concentre à lui seul plus de la moitié des paroisses concernées. Il est intéressant de noter à cette occasion que, de longue date, le chapitre disposait librement de 40 cures qu'il pouvait confier à qui bon lui semblait ${ }^{60}$. Mais les cures que cumulent les chanoines de la cathédrale ne sont pas celles-ci. Cela signifie que le chapitre contrôlait directement non seulement les cures à sa collation mais aussi celles que possédaient ses membres. En plus des juridictions décanales et archidiaconales, le chapitre disposait donc d'un contrepoids important aux volontés de l'évêque et cela explique très certainement les conflits entre l'évêque et son chapitre qui ont émaillé le XIII ${ }^{\mathrm{e}}$ siècle et le début du XIV ${ }^{\mathrm{e}}$ siècle $^{61}$. La présence de chanoines disposant d'églises paroissiales n'est pas exceptionnelle et n'était pas interdite. En effet, les prébendes de cathédrale n'ayant pas de charge d'âmes pouvaient être cumulées avec des cures paroissiales. De plus, la résidence mancelle n'empêchait nullement les chanoines de la cathédrale d'effectuer leur ministère dans leur cure. Ainsi, en 1355, six chanoines cumulant prébendes et églises demandent une dispense de résidence pour assurer leur charge paroissiale ${ }^{62}$. L'attribution d'une église est souvent antérieure à l'obtention d'un canonicat; toutefois Jean de Baugé, attesté comme chanoine en 1349, ne reçoit l'église paroissiale de Grysello ${ }^{63}$ qu'en 1359. La présence au chapitre a certainement joué dans son obtention.

58. 11 chanoines ont disposé de deux églises paroissiales et deux en ont eu trois.

59. BRION, Isabelle de, Les chanoines de la cathédrale d'Angers..., op. cit., p. 81.

60. Cette situation a été confirmée en 1313 par l'évêque Pierre Gougeul (CAUvin Thomas, Géographie ancienne du diocèse du Mans, Paris/Le Mans, Derache, Galienne, 1845, p. XCI).

61. Le texte, cité ci-dessus, est lui-même la conséquence d'un conflit de juridiction entre Pierre Gougeul et son chapitre sur ces quarante cures (voir Annexe. Carte du diocèse du Mans).

62. Médiathèque du Mans, ms. 247, $\mathrm{f}^{\circ} 6$.

63. Archivum Secretum Vaticanum, Registre d'Avignon n ${ }^{\circ} 106, \mathrm{f}^{\circ} 657 \mathrm{Je}$ n'ai pas réussi à traduire le nom de cette cure du diocèse, plusieurs paroisses s'en rapprochant comme Grez ou Grées. 
Le Mans n'a pas été le seul diocèse à être marqué par le cumul d'églises paroissiales. Les diocèses du Sud constituent d'ailleurs des cas assez exceptionnels, avec un personnage comme Pierre Raffin, chapelain d'un cardinal. Ce chanoine du Mans, de 1316 à 1324, cumulait, semble-t-il, pas moins de six églises paroissiales ${ }^{64}$ ! Il est difficile de dire si ce cumul était réel ou s'il a reçu les bénéfices successivement. En effet, en 1316, il dispose de deux églises paroissiales qu'il ne semble plus posséder en 1324. Une chose est sûre, lors de son décès vers le mois de mai 1324, ses bénéfices, quatre églises dans les diocèses de Narbonne et de Rodez, sont redistribués. Il a, de toute manière, disposé de bénéfices de manière "illégale ", que ce soit en 1316 où le cumul était interdit ou en 1324 où le type de cumul qu'il pratiquait, était illicite.

Le cumul des églises paroissiales est donc une réalité au sein du chapitre de la cathédrale du Mans. Par ailleurs, le cumul de chapellenies existait également mais ne touchait que 37 bénéfices dont la plupart dans les diocèses du Mans (14) et d'Angers (13).

Le cumul des bénéfices au sein du chapitre cathédral du Mans a donc connu une extension géographique très importante, grâce essentiellement au cumul de prébendes cathédrales qui est celui qui concerne le plus de chanoines et qui couvre la plus grande aire géographique. Le cumul a permis de créer un réseau de relations pour le chapitre dans les autres diocèses de France et de l'étranger même les plus éloignés. Cependant, il est impossible d'affirmer que la recherche d'une prébende mancelle pour les cumulateurs du Sud de la France a été la préoccupation première. La recherche d'une prébende cathédrale paraît répondre à un besoin financier et on ne voit pas quelles raisons particulières auraient poussé ces clercs à rechercher des prébendes au Mans plus qu'ailleurs; la quête d'une riche prébende en France du Nord a dû être la préoccupation centrale. La préoccupation des chanoines des diocèses du Nord paraît également financière, au moins pour ceux qui disposent de plus d'une prébende. Pour les autres, le chapitre cathédral du Mans apparaît comme une " position de repli " assez peu éloignée de leur région d'origine en cas d'échec dans la demande auprès de la cour pontificale d'un bénéfice dans d'autres chapitres cathédraux.

\section{Les cumulateurs au sein du chapitre}

Le cumul de la part des Manceaux s'effectuait donc dans de nombreuses régions de l'Occident chrétien. Cette extension pose la question de la relation qu'entretenaient les chanoines avec « leur " chapitre ainsi que celui de la résidence dans les bénéfices.

64. Trois dans le diocèse de Rodez, deux dans le diocèse de Narbonne et une dans le diocèse d'Albi. 


\section{Des canonicats d'une durée habituelle}

Le nombre de chanoines cumulant des bénéfices n'a pas connu beaucoup de variations dans le temps. Le nombre moyen de chanoines cumulateurs entre 1294 et 1378 est d'à peu près 12 par an. Le maximum est atteint en 1371 avec 22 personnes. Globalement, l'évolution du nombre de cumulateurs suit celle de l'effectif de chanoines connus. Cependant, les années pour lesquelles ceux-ci sont les plus nombreux sont celles pour lesquelles la proportion de cumulateurs est souvent la plus faible. Ainsi, pour l'année 1309, 37 chanoines sont connus pour seulement 10 cumulateurs et, pour l'année 1346, les cumulateurs représentent une petite moitié (16 personnes) des 37 chanoines. Les proportions restent constamment inférieures à la moitié du total. Pour les années les moins fastes du début de la période, entre 1294 et 1308, les proportions sont bien différentes. C'est ainsi qu'en 1302, sur 9 chanoines connus, 8 cumulent plusieurs bénéfices. Cette disparité s'explique par la documentation; en effet, avant 1309, les sources mancelles sont assez rares et face à ce manque, il est nécessaire de faire appel aux sources pontificales qui sont plus développées pour ce qui est des bénéfices dont disposent les clercs.

La durée d'un canonicat pendant cette période est assez difficile à connaître et cela oblige à faire des estimations. En effet, nous ignorons souvent les dates précises de début et de fin de canonicat mais il existe des "dates butoirs" car certaines sources fournissent la presque totalité des noms pour une date précise. Les chanoines non présents dans ces listes mais présents dans des sources antérieures ont nécessairement cessé d'être chanoine du Mans avant la constitution des listes en question. À partir de ces données, il apparaît qu'entre 1294 et 1378, la durée moyenne d'un canonicat à la cathédrale du Mans est de six ans et demi, avec un minimum d'un an et un maximum de cinquante-quatre ans. Pour les chanoines qui cumulent des bénéfices la moyenne est de huit ans, avec un écart tout aussi important. Les canonicats qui ne durent qu'un an sont assez nombreux et représentent $35 \%$ des canonicats de chanoines cumulateurs. Cette forte proportion est certainement due aux lacunes de la documentation. Par exemple, entre 1294 et 1309, les listes complètes n'existent pas, certaines personnes ont donc très bien pu être chanoines pendant cette période mais elles n'apparaissent qu'en 1309 et ont pu décéder ensuite avant la liste complète établie vers $1316^{65}$. D'autre part, certains chanoines pourvus apparaissent pour un an seulement dans les sources mancelles, du fait de la dispense de résidence. Quoi qu'il en soit, cette moyenne est intéressante et nous révèle que la prébende mancelle, malgré le cumul d'autres bénéfices, ne passe pas de main en main sans arrêt. D'ailleurs le chanoine dont le canonicat semble le plus long (54 ans)

65. BERTRAND de Broussillon, Arthur, Cartulaire..., op. cit., $\mathrm{n}^{\circ} 1116$. Cette liste récapitule tous les membres du chapitre, y compris le roi de France et les abbés de Saint-Vincent et Saint-Calais. Elle classe les chanoines selon leur ordre religieux (d'abord les prêtres, ensuite les diacres...). L'inconvénient est qu'elle ne donne pas les noms des dignitaires. 
est Renaud Pansé, qui a cumulé plusieurs bénéfices. Il existe certainement un attachement à cette prébende malgré un cumul parfois très développé. Les quelques cardinaux qui ont disposé d'une prébende l'ont, pour la plupart, conservée jusqu'à leur décès. Cet attachement s'explique relativement bien pour les clercs manceaux qui conservent un bénéfice dans leur diocèse d'origine mais pour les autres cumulateurs, le problème reste entier.

\section{Des dignitaires cumulateurs}

Si la majorité des cumulateurs ont été simplement chanoines au Mans, certains ont exercé des fonctions plus importantes. Les cumulateurs du chapitre ont été nombreux à occuper ces postes; ainsi, 7 doyens sur 10 ont cumulé plusieurs bénéfices ${ }^{66}$. Certains sont d'anciens membres du chapitre devenus doyen par la suite. Cependant, trois seulement sont issus de manière sûre du chapitre. Le décanat de l'église du Mans est, somme toute, un bénéfice comme un autre, qui peut être cumulé bien que, d'après la constitution de 1317, il soit soumis à des restrictions. Le décanat est en fait pour certains le sommet d'une carrière, avant qu'ils ne soient promus à un évêché. Cette dignité semble à la fois source de revenus et de prestige; en effet, aucun des doyens cumulateurs ne dispose de bénéfices plus importants que celui-ci. Ainsi, Gilbert Hamelin, pourvu en 1329, en plus d'être médecin des rois de France, n'était que chanoine de Paris ou de Chartres. Sur l'ensemble de la période la présence des doyens cumulateurs aux chapitres témoigne d'un intérêt, au moins lointain, pour " leur " chapitre. Dans la plupart des réunions du chapitre, le doyen est présent, sauf en 1312 où Pierre Gougeul (l'ancien doyen) venait tout juste d'être nommé évêque du Mans.

Les archidiacres sont les plus représentés parmi les dignitaires cumulateurs. Ils sont au nombre de 27 , sur 40 archidiacres connus pour toute la période. Leur cas est un peu particulier car pour la plupart ils sont des cardinaux. L'archidiaconé du Mans, après avoir été détenu par Jean de Tocco, passe de main en main au sein de la curie, d'abord avec Bertrand du Pouget (après 1316), puis avec Nicolas Capocci (1357-1368), et enfin avec Jean de Dormans (1368-1373). Pour cette période, seul le dernier archidiacre, Denis Jean, n'était pas cardinal. Sur les 6 archidiacres du Mans, les deux seuls non cardinaux sont tout de même des cumulateurs ${ }^{67}$. L'intérêt pour cet archidiaconé paraît financier : il se trouve en effet être le plus rentable

66. Les doyens du Mans connus pour la période 1294-1378 sont : Robert de Clinchamp (1285-1300), Pierre Gougeul (1300-1312), Jean de Chambre (1314-1322), Jean de Vienne (1322-1328), Gilbert Hamelin (après 1329), Mathieur Pezas (avant 1346-1348), Guillaume de Rochefort (après 1348), Robert Hélie (vers 1362-1370) et Laurent Baulanié (1370-1383).

67. Jean de Tocco (1307-1315) était chanoine de Saint-Jacques de Barletta et Denis Jean (archidiacre jusqu'en 1375) disposait de l'église paroissiale de Grazay, dans le diocèse du Mans. 
de tous ${ }^{68}$. Le phénomène se reproduit bien pour les autres archidiaconés mais les cardinaux sont absents de ces derniers. La forte proportion d'archidiacres cumulateurs explique leurs absences aux réunions du chapitre, ce qui pose le problème de la résidence de ces dignitaires qui avaient une juridiction sur une partie du diocèse, et ceci a dû faire perdre une partie de son pouvoir au chapitre face à l'évêque. Le XIV ${ }^{\mathrm{e}}$ siècle est d'ailleurs une période de récupération de ses prérogatives par ce dernier. Vers 1380 certains archidiacres signent des actes de renonciations à leur juridiction dans les archidiaconés hormis les visites ${ }^{69}$.

Les archidiacres ne sont pas les seuls concernés par le cumul. Il touche toutes les dignités du chapitre : 2 scolastiques, 3 chantres et 1 sous-chantre, mais dans des proportions moindres à cause du faible nombre de ces personnages dans le chapitre pendant la période ( 2 scolastiques et 11 chantres et sous-chantres).

La présence de chanoines et dignitaires cumulateurs au sein du chapitre cathédral du Mans est donc une réalité assez prégnante. Ces chanoines ne semblent pas être très différents de leurs collègues et assument des dignités "trustant " même les archidiaconés. Ce phénomène pose, tout de même, le problème de la résidence de ces personnages dans le chapitre et par-là même de leur implication réelle dans la vie de celui-ci.

\section{Le problème de la résidence}

La non-résidence est un problème assez récurrent dans les chapitres séculiers au Moyen Âge ${ }^{70}$ et Le Mans ne semble pas échapper à la règle. La possession de bénéfices à l'extérieur du diocèse pose de fait la question de savoir si les chanoines résidaient dans leurs bénéfices manceaux ou bien dans leurs autres bénéfices. Il est assez complexe d'étudier la présence des chanoines au Mans, même s'il existe des listes de noms pour certaines décisions capitulaires (1309, 1312, 1314, 1346, 1350, années 1370). Pour le début de la période étudiée, le taux de participation des chanoines aux chapitres est d'un peu plus de $50 \%$. Le cas des chanoines cumulateurs est équivalent. En 1309, le chapitre général enregistre le record de présences avec 36 personnes. Il ne manque en effet que l'archidiacre de Sablé (Robert de Malo Vicino), un cumulateur. Pour cette année-ci, le nombre potentiel de chanoines cumulateurs est de neuf, dont huit sont présents. En 1312, vingt-deux chanoines assistent à la réunion, soit un peu moins de la moitié de l'ensemble des chanoines. Deux cumulateurs sur huit sont présents mais

68. Longnon, Auguste, Pouillés..., op. cit., p. 58. L'archidiaconé du Mans et celui de Sablé sont taxés à hauteur de 4 livres contre 60 à 70 sous pour les autres archidiacres.

69. Cette renonciation est avérée pour les archidiacres de Montfort et du Mans (Médiathèque du Mans, ms. 247, $\mathrm{f}^{\circ}$ 45-46).

70. Hélène Millet l'évoque dans sa thèse sur Laon. Voir aussi MATZ, Jean-Michel, " Des stalles vides au chœur? La résidence des chanoines d'Angers a la fin du Moyen Age ", dans Billiet, Frédéric (dir.), Bible de bois du Moyen Âge-Bible et liturgie dans les stalles médiévales, Angers, Université catholique de l'Ouest, Paris, L'Harmattan, 2004, p. 17-36. 
ils étaient déjà là en 1309. Pour 1314, la situation est presque identique ${ }^{71}$; seule originalité, l'archidiacre du Mans, Jean de Tocco, se fait représenter par un procureur, Jean de Canale (recteur de l'église paroissiale de Sargé, diocèse du Mans).

Entre 1314 et 1346, nous ne disposons plus de délibérations capitulaires mais seulement d'un registre qui indique les noms des chanoines payant un loyer pour une maison ou des propriétés du chapitre ${ }^{72}$. Le nombre global de bénéficiaires de ces propriétés au Mans durant ce laps de temps est de 18 à 21. Pour toutes les années de la période, les cumulateurs représentent en moyenne le tiers des personnes payant un loyer pour une maison. Les cumulateurs ne semblent donc pas moins impliqués dans la vie du chapitre que la moyenne des autres chanoines. Il est difficile de dire si les personnes résidaient ou non. Une partie des « locataires " résidaient sûrement au Mans mais la proportion n'est pas connue.

À partir de 1346, des listes réapparaissent mais sont rares (1346, 1350, 1377). La réunion de 1346 fournit la plus importante liste conservée depuis 1309 (23 présents). Les chanoines cumulateurs pour cette même année étaient au nombre de 17. Neuf d'entre eux ont fait le déplacement au chapitre. Un peu moins de la moitié des cumulateurs se déplace en 1350 (6 sur 16) et pour la réunion datée de 1377 (7 sur 17) ${ }^{73}$.

Durant l'ensemble du XIV ${ }^{\mathrm{e}}$ siècle, il semble que les chanoines cumulateurs soient tout aussi assidus aux chapitres que leurs homologues non cumulateurs. Cela s'explique assez bien étant donné que les chanoines présents cumulent pour la plupart leurs bénéfices au Mans ou dans le diocèse. Le cumul n'impliquait donc pas nécessairement la non-résidence. En effet, si des personnages cumulaient des bénéfices au Mans ou dans le diocèse, ils pouvaient résider au Mans ou dans leurs autres bénéfices, sans que cela ait des conséquences néfastes sur les autres bénéfices cumulés.

Par contre, la résidence dans des régions plus ou moins lointaines suppose, de fait, l'absentéisme dans les autres diocèses (Le Mans inclus). À l'inverse, si les cumulateurs résidaient au Mans, la non-résidence a nécessairement eu des conséquences dans les autres bénéfices concernés par le cumul. Il est malheureusement difficile, faute d'études locales, d'évaluer ces conséquences, partout où les chanoines du Mans ont eu des bénéfices. Pour ce qui est des églises paroissiales, la pratique du cumul ne signifie pas cependant l'abandon total de cette église une fois reçu un autre bénéfice

71. Le chapitre général du mois de juin 1314 regroupe 22 chanoines dont 3 cumulateurs pour 8 potentiels. BERTRAND de Broussillon, Arthur, Cartulaire..., op. cit., $\mathrm{n}^{\circ} 1114$.

72. Arch. dép. de la Sarthe, G 964. Il s'agit d'un livre de comptes qui commence au mois de juin 1337 et se termine en juin 1346.

73. BERTRAND de BroussiLlon, Arthur, Cartulaire..., op. cit., n 1173,26 novembre 1350. La liste donnée à la fin du texte de 1377 est probablement en partie une copie d'autres listes précédentes. En effet, on y retrouve Simon de Vares, scolastique à partir de 1356, alors que ce dernier est décédé vers 1375. Cette liste est malgré tout intéressante car elle est tout de même postérieure et de nouveaux noms apparaissent. 
plus important. Le dépositaire du bénéfice avait donc recours à des représentants pour maintenir l'activité pastorale dans les paroisses. Raoul de La Roche en est d'ailleurs un bon exemple car il a été chanoine entre 1363 et 1371 et vicaire d'une église paroissiale (c'est le seul dans ce cas) du diocèse de Castres. Lors de sa résignation en 1371, il dispose d'une église paroissiale dans ce même diocèse. Il est impossible d'affirmer qu'il a été curé en même temps que vicaire, ce qui est peu probable; cependant il est intéressant de noter qu'il reproduit son expérience de vicaire car il ne pouvait pas se trouver dans tous ses nombreux bénéfices à la fois, bien que tous soient situés dans le diocèse de Castres. Le recours à des vicaires au moins pour les bénéfices les moins importants était une nécessité; comme l'exprime Hélène Millet dans sa thèse, le développement du cumul des églises paroissiales a entraîné en parallèle le développement d'une catégorie de clercs chargés de la vie quotidienne des paroisses qui ne rentrait pas dans le jeu du cumul et qui était de plus contrainte à la résidence ${ }^{74}$. S'ils résidaient au chapitre, les chanoines du Mans ont ainsi pu développer une " clientèle ", dans leur diocèse d'origine.

Le cumul des bénéfices est donc une réalité incontestable au sein du chapitre cathédral du Mans. Ce cumul s'exerce sur l'ensemble du territoire français mais aussi à l'étranger. Cela a sans doute permis au chapitre de constituer un réseau de relations dans les divers diocèses et chapitres concernés. Par ailleurs, les cumulateurs apparaissent comme une catégorie à part au sein du chapitre. Si leur cumul a entraîné une non-résidence, cela a permis au chapitre de disposer d'un important réseau d'appuis auprès des institutions tant royales que pontificales. Ces personnages ont donc certainement contribué à servir les intérêts du chapitre. Enfin, le cumul dans le chapitre ne semble pas avoir eu de conséquences trop néfastes sur la vie de celui-ci. La vie quotidienne de la communauté devait se maintenir grâce à la présence de chanoines non-cumulateurs ou de chanoines cumulateurs qui ne disposaient pas de bénéfices lointains.

Les motivations des cumulateurs ressortent difficilement dans le cadre de cette étude. Pour les clercs du diocèse du Mans, le canonicat de la cathédrale semble être l'aboutissement d'une carrière. Cela conférait aux clercs prestige et ressources financières tout en leur permettant de conserver leurs bénéfices précédents. Pour les serviteurs des institutions extérieures, cela apparaît davantage comme un complément aux revenus versés par leurs maîtres ou patrons qui y voyaient eux aussi leur intérêt. Ces clercs pouvaient être aussi de bons relais de l'autorité de leurs maîtres. Enfin pour les grands cumulateurs des familles nobles, c'est la solution pour assurer un moyen de subsistance à des cadets consacrés à l'Église.

L'absence d'autres études spécifiques sur les cumulateurs limite les possibilités de comparaison. Les cas de cumul sont presque aussi nom-

74. MilLET, Hélène, Les Chanoines de Laon..., op. cit., p. 227. 
breux que les cumulateurs eux-mêmes. Même s'il existe des catégories de cumulateurs, il n'y a pas de portrait-type, chacun cumulant dans une ou plusieurs régions des types de bénéfices parfois divers. Malgré tout, les chanoines cumulateurs paraissent composer une élite au sein d'une institution capitulaire, qui en est elle-même une au niveau de l'Église diocésaine.

\section{RÉSUMÉ}

Cet article traite du cumul de bénéfices ecclésiastiques par les chanoines de la cathédrale du Mans au XIV'e siècle. Il s'agit d'une étude concernant tous types de bénéfices : prébendes et dignités de cathédrales, de collégiales et églises paroissiales. Après une rapide évocation de l'organisation du chapitre cathédral du Mans à cette époque, ce travail analyse les différents aspects de la pratique du cumul. Cela porte tout d'abord sur les bénéfices cumulés, puis sur les chanoines qui pratiquaient le cumul. L'étude des chanoines cumulateurs recouvre autant leur origine sociale et géographique que leurs activités à l'extérieur du chapitre : service de la cour pontificale, de seigneurs laïques, d'évêques et enseignement. Enfin l'influence du cumul sur la vie du chapitre cathédral est étudiée : durée des canonicats, relation des dignitaires du chapitre cathédral du Mans au cumul et présence des cumulateurs dans le quotidien de l'institution.

\section{ABSTRACT}

This article deals with the canons of the cathedral of Le Mans and plurality of benefices in the 14th century. It is a study about all types of benefices: prebends and dignities of cathedrals, collegiate churches and parochial churches. After a quick evocation of the organisation of the cathedral chapter of Le Mans in this period, this work analyses the various aspects of the plurality of benefices. It first of all examines the benefices, then the canons who occupied these positions. The study of these canons encompasses their social and geographical origins as well as their activities outside the chapter such as serving the papal court, lay Lords, bishops or teaching. Finally, the impact of the plurality of benefices on the life of the cathedral chapter is studied through an analysis of the duration of the canonries, the relationship of the dignitaries of the cathedral chapter of Le Mans to the plurality of benefices and the presence of the holders of these positions in the daily life of the institution. 